\title{
Descripción de la experiencia en pacientes diagnosticados con insulinoma. Estudio multicéntrico en Medellín, Colombia
}

\author{
Catalina Tobón-0spina', Pablo Castaño', Johnayro Gutiérrez-Restrepo ${ }^{2}$, José Luis Torres-Grajales', \\ Sergio Iván Hoyos-Duque ${ }^{4}$, Juan Camilo Pérez-Cadavid 5 , Jorge Hernando Donado-Gómez ${ }^{6}$, Alejandro Román-González 2,7
}

\section{RESUMEN}

Objetivo: describir la experiencia de los pacientes con insulinoma, diagnosticados y tratados entre los años 2002 y 2017 en tres hospitales de la ciudad de Medellín, Colombia.

Métodos: estudio descriptivo y retrospectivo de pacientes con criterios bioquímicos para hipoglucemia hiperinsulínica y confirmación histopatológica de insulinoma.

Resultados: se estudiaron 19 casos, 10 eran mujeres (52,6 \%), la edad media al diagnóstico fue 43 años (D.E 15,5). Hubo cuatro casos de insulinoma multifocal (21,1\%), tres asociados con NEM-1 (15,8 \%) y dos malignos (10,6 \%). Todos presentaron hipoglucemia en ayunas y $63,2 \%$ posprandial. En la prueba de ayuno, el nadir de glucemia sucedió antes de 48 horas en todos los casos, en promedio 9 horas (D.E 8,0). El diagnóstico bioquímico fue realizado con hipoglucemia e insulina elevada en todos los casos, aunque el péptido $C$ fue reportado en nueve pacientes $(47,3 \%)$ y las sulfonilureas en dos $(11,1 \%)$. La localización preoperatoria se hizo por imágenes en 12 individuos (68,5 \%) y las pruebas invasivas fueron necesarias en seis (31,5\%). Las pruebas diagnósticas fueron positivas en un $83 \%$ para resonancia, $50 \%$ para ecografía endoscópica y prueba de estímulo intraarterial con calcio y $100 \%$ para ecografía intraoperatoria. La ciruớa se realizó en 18 casos (94,7 \%). La mortalidad (15,8 \%) fue derivada de complicaciones en el posoperatorio temprano; la curación se logró en todos los casos.

Conclusiones: el insulinoma en nuestro medio tiene características demográficas y clínicas similares a otras series. Existen limitaciones locales para el acceso a los estudios bioquímicos

\footnotetext{
1 Médico Internista, Residente de Endocrinología Clínica y Metabolismo, Universidad de Antioquia, Medellín, Colombia.

Internista Endocrinólogo Profesor Departamento de Medicina Interna, Universidad de Antioquia, Antioquia, Medellín, Colombia.

3 Especialista en Endocrinología Clínica, Clínica Las Américas, Medellín, Colombia. Profesor Universidad Pontifica Bolivariana.

4 Cirujano Hepatobiliar y de trasplantes, Hospital Pablo Tobón Uribe. Profesor Universidad de Antioquia, Medellín, Colombia.

5 Patólogo, Hospital Pablo Tobón Uribe, Medellín, Colombia.

6 Internista. Jefe Unidad de Investigaciones y Docencia Hospital Pablo Tobón Uribe. Profesor Universidad de Antioquia, Medellín, Colombia.

7 Internista Endocrinólogo Hospital Universitario San Vicente Fundación, Medellín, Colombia.
}

Correspondencia: Alejandro Román-González; alejoroman@gmail.com

Recibido: 23 de mayo de 2019

Aceptado: 22 de agosto de 2019

Como citar: Tobón-0spina C, Castaño P, Gutiérrez-Restrepo J, Torres-Grajales JL, Hoyos-Duque SI, Pérez-Cadavid JC, et al. Descripción de la experiencia en pacientes diagnosticados con insulinoma. Estudio multicéntrico en Medellín, Colombia. latreia. 2020 Abr-Jun;33(2):133-142. D0I 10.17533/udea.iatreia.41. 
y en el rendimiento diagnóstico de las pruebas de localización.

\section{PALABRAS CLAVE}

Hiperinsulinismo; Hipog̊lucemia; Insulinoma; Páncreas; Tumores Neuroendocrinos

\section{SUMMARY}

Description of the experience with patients with insulinoma diagnosis. Multicentric study in Medellín, Colombia

Objective: The objective of the study was to describe the characteristics of patients with insulinoma in three hospitals in Medellín, Colombia, between 2002 and 2017.

Methods: A retrospective analysis of patients with hyperinsulinemic hypoglycemia and histologic confirmation of insulinoma was conducted.

Results: A total of 19 cases were identified. Ten women $(52.6 \%)$ and 9 males $(47.4 \%)$. The mean age at diagnosis was 43 years (S.D: 15.5). Four cases had multifocal insulinoma (21.1\%), 3 cases were secondary to multiple endocrine neoplasia type $1(15.8 \%)$, and 2 of them were maliognant $(10.6 \%)$. All patients presented fasting hypoglycemia, and $63.2 \%$ presented post-prandial hypoglycemia. Glucose nadir in the fasting test occurred in the first 48 hours in all cases, with a mean time to hypoglycemia of 9 hours (S.D 8.0). The biochemical diagnosis was done with increased insulin in the presence of hypoglycemia. C-peptide was done in 9 patients (47.3\%), and sulfonylureas in 2 cases (11.1\%). Preoperative localization was done by imaging in 12 cases (68.5\%), and invasive tests were required in 6 cases ( $31.5 \%$ ). Localization tests were positive as follows: magnetic resonance imaging in $83 \%$, endoscopic ultrasound in 50\%, selective intra-arterial calcium injection in $50 \%$, and intraoperative ultrasound in $100 \%$. Eighteen patients (94. $7 \%$ ) underwent surgery. Mortality (15.8\%) was secondary to early post-operative complications.

Conclusions: The characteristics of patients with insulinoma in Medellín are similar to other series.
However, there are important local limitations for proper biochemical testing and imaging localization. This is the largest study in our country.

\section{KEYWORDS}

Hyperinsulinism; Hypoolycemia; Insulinoma; Neuroendocrine Tumors; Pancreas

\section{INTRODUCCIÓN}

EI insulinoma es un tumor pancreático secretor de insulina. A pesar de su baja incidencia, estimada en cuatro casos por cada millón de personas al año, constituye la primera causa de hiperinsulinismo endógeno en adultos ${ }^{(1,2)}$. La mayoría de los insulinomas son tumores benignos, solitarios y esporádicos ${ }^{(3)}$. La hgipoglucemia es la manifestación clínica cardinal y la responsable de la morbilidad ocasionada por el tumor ${ }^{(4)}$. Para el diagnóstico es necesario documentar hiperinsulinismo mediante la prueba de ayuno de 72 horas o durante un episodio de hipoglucemia espontánea. El perfil bioquímico se caracteriza por hipoglucemia con insulina y péptido C elevados, excluyendo los diagnósticos alternativos como la administración exógena de sulfonilúreas ${ }^{(2,5)}$. Luego se procede a localizar el tumor por técnicas invasivas o no invasivas. El tratamiento de elección es quirúrgico ${ }^{(5)}$.

La rareza relativa de esta entidad explica la escasez de reportes locales en la literatura. Los estudios publicados en Colombia están limitados a reportes con un número pequeño de casos y procedentes de un solo centro hospitalario ${ }^{(6-9)}$. Se desconoce en la actualidad la experiencia local en relación con el diagnóstico y tratamiento de esta entidad. El objetivo del presente estudio fue evaluar de forma retrospectiva la experiencia clínica de los pacientes con insulinoma en tres hospitales de alta complejidad en la ciudad de Medellín, Colombia.

\section{PACIENTES Y MÉTODOS}

Estudio observacional, ambispectivo de serie de casos, en pacientes con insulinoma, diagnosticados 
entre los años 2002 y 2017 en tres instituciones de la ciudad de Medellín: Hospital Pablo Tobón Uribe (HPTU), Hospital San Vicente Fundación (HSVF) y Clínica Las Américas. Se incluyeron individuos mayores de 14 años con criterios bioquímicos e histopatológicos de insulinoma. Se excluyeron aquellos con hipoglucemia secundaria a otras causas y demás con resección quirúrgica realizada fuera de Ios hospitales de referencia del estudio. Se registraron variables clínicas, demográficas, paraclínicas y de imágenes para cada caso. Se revisó la patología del tumor y se registró el número de tumores, tamaño, índice mitótico, índice de proliferación (Ki-67), inmunohistoquímica y clasificación de la Organización Mundial de la Salud 2017 (si estaban disponibles todos los datos).

Esta clasificación divide a los tumores neuroendocrinos pancreáticos según su grado de diferenciación: tumores bien diferenciados y mal diferenciados $y$, a su vez, los tumores bien diferenciados, se subdividen en tres grados histológicos según el Ki67 y la tasa mitótica. Así, los grado I son aquellos con un ki67 menor a 3 \% y menos de 2 mitosis por 50 campos de alto poder (CAP); los grado II son aquellos con un Ki67 entre 3-20\% o 2-20 mitosis por CAP y; los grado III aquellos con un Ki67 mayor al $20 \%$ o más de 20 mitosis por CAP.

Se definió curación como ausencia de hipoglucemia hasta seis meses después de la ciruogía, se realizó una llamada telefónica, con previo consentimiento informado, para verificar el estado vital. La llamada telefónica fue realizada prospectivamente, el estado vital fue confirmado por el equipo investigador $y$ no estaba consignado necesariamente en la historia clínica.

\section{Análisis estadístico}

Las variables cuantitativas se expresaron como medias \pm desviación estándar (D.E) si presentaban distribución normal o, como medianas y rango intercuartílico (RIC), si la distribución era no paramétrica. Las variables cualitativas se expresaron como porcentaje. La normalidad de las variables cuantitativas se calculó mediante la prueba de Shapiro-Wilk. Los análisis estadísticos se realizaron mediante el programa SPSS IBM, versión 22.

\section{Aspectos éticos}

Este estudio fue aprobado por el comité de ética de cada hospital y la aprobación del protocolo por la Universidad de Antioquia. Los investigadores se adhirieron a la declaración de Helsinki (versión 2013) según la regulación colombiana: Resolución No. 8430 de 1993.

\section{RESULTADOS}

\section{Características demográficas y clínicas}

Se estudiaron 19 pacientes: 13 del HPTU (68,4\%), 5 del HSVF $(26,4 \%)$ y uno de la Cínica Las Américas (5,2 \%). Diez eran mujeres $(52,6 \%$ ) y 9 hombres $(47,4 \%)$, la razón mujer/hombre fue de 1:1. El promedio de edad al diagnóstico fue 43 años (D.E: 15,5). La mediana en meses del tiempo de evolución de los síntomas al diagnóstico fue de 11 meses (RIC: 3-23). El antecedente familiar de NEM-1 (neoplasia endocrina múltiple-1) estuvo presente en un caso $(5,2 \%) y$, el antecedente de tumores neuroendocrinos en familiares de primer grado estuvo presente en dos pacientes (10,5\%). Diecisiete individuos presentaron síntomas neuroglucopénicos (89,4\%), 13 individuos, síntomas simpáticos $(68,4 \%$ y y siete ganaron peso $(36,8 \%)$. En siete personas existió un diagnóstico inicial erróneo alterno a hipoglucemia (36,8\%), el más frecuente de ellos fue la epilepsia $(n=6 ; 31,5 \%)$, seguido del trastorno psiquiátrico ( $\mathrm{n}=1 ; 5,3 \%$ ).

No se reportó el patrón de la hipoglucemia en el 57,8 \% de los pacientes $(\mathrm{n}=11)$. De los patrones reportados, 7 (36,8 \%) fueron hipoglucemias durante el estado de ayuno y, el restante (5,4\%), fue un patrón mixto, ayuno y posprandial.

En la presente serie hubo 16 casos de insulinoma esporádico $(84,2 \%)$ y tres asociados con NEM-1 (15,8\%); todos los casos con NEM-1 presentaron insulinomas multifocales. Hubo 17 casos de insulinoma benigno $(89,4 \%)$ y otros dos de insulinoma maligno $(10,6 \%)$, ambos con metástasis hepáticas múltiples.

En relación con la multiplicidad del tumor, hubo 15 casos de insulinoma único $(78,9 \%)$ y cuatro de insulinoma multifocal $(21,1 \%)$. Uno de los casos de insulinoma único correspondió a un insulinoma maliogno sin intervención quirúrogica, en la cual el diagnóstico de la lesión primaria fue realizado solo por imágenes. 


\section{Estudios bioquímicos}

La prueba de ayuno (72 horas) se realizó en 11 pacientes $(57,8 \%)$. Los siete casos restantes fueron diagnosticados durante la hipoglucemia espontánea (36,8 \%). En un caso se desconoce el método para el diaognóstico bioquímico inicial, debido a que los estudios fueron en otra institución. En todos Ios individuos diagnosticados por prueba de ayuno, el nadir de glucemia se presentó antes de 48 horas, en promedio 9 horas, (D.E: 8,0). Los valores de la glucemia plasmática basal, insulinemia y péptido $C$ se muestran en la (Tabla 1). Los niveles de insulina y péptido $C$ en todos los pacientes incluidos alcanzaron el valor diagnóstico para hiperinsulinismo endógeno de acuerdo con los criterios establecidos por las guías ENETS (Sociedad Europea de Tumores Neuroendocrinos). El nivel de glucemia más bajo fue $22 \mathrm{mg} / \mathrm{dL}$, con valores correspondientes de insulina de $27,4 \mu \mathrm{U} / \mathrm{mL}$ y péptido $C 3,7 \mathrm{ng} / \mathrm{mL}$. La prueba de glucagón no fue realizada en ninguna de las instituciones. La cetonemia fue negativa en los casos medidos $(n=7)$. Se realizó la medición de sulfonilureas en dos personas, con reporte negativo en ambos casos. La medición de anticuerpos antiinsulina no se efectuó en ningún caso.

Tabla 1. Características demográficas con los valores de laboratorio

\begin{tabular}{ccccccc}
\hline Caso & Sexo & Edad & Evolución (meses) & $\begin{array}{c}\text { Glucemia } \\
(\mathbf{m g} / \mathbf{d L})\end{array}$ & $\begin{array}{c}\text { Insulina } \\
(\boldsymbol{\mu U} / \mathbf{m L})\end{array}$ & $\begin{array}{c}\text { Péptido-C } \\
(\mathbf{n g} / \mathbf{m L})\end{array}$ \\
\hline 1 & M & 34 & 2 & 27 & 16,8 & 2,6 \\
\hline 2 & M & 27 & 7 & 39 & 23 & 3,9 \\
\hline 3 & M & 30 & 35 & 25 & 236 & 15,1 \\
\hline 4 & M & 62 & 3 & 40 & 9,5 & 5,6 \\
\hline 5 & F & 51 & 3 & 22 & 27,4 & 3,7 \\
\hline 6 & F & 53 & 24 & $*$ & $*$ & $*$ \\
\hline 7 & F & 52 & 52 & 33 & 5.9 & $*$ \\
\hline 8 & M & 35 & 3 & 41 & 10,9 & $*$ \\
\hline 9 & F & 40 & 12 & 36 & 6,7 & 2,39 \\
\hline 10 & F & 71 & 18 & 53 & 9,1 & $*$ \\
\hline 11 & F & 33 & 24 & 38 & 9,8 & $*$ \\
\hline 12 & F & 15 & 8 & 19 & 81,8 & 3 \\
\hline 13 & H & 30 & 60 & 34 & 8,2 & $*$ \\
\hline 14 & M & 45 & 10 & 35 & 14,2 & $*$ \\
\hline 15 & H & 35 & 1 & 47 & 24,2 & 4,5 \\
\hline 16 & H & 62 & 2 & 54 & 19,8 & $*$ \\
\hline 17 & M & 33 & 12 & 45 & 21,9 & $*$ \\
\hline 18 & H & 69 & 48 & & $*$ & $*$ \\
\hline 19 & M & 53 & $*$ & 29 & 72 & 3,8 \\
\hline M & & & & & & $*$ \\
\hline
\end{tabular}

M: mujer. H: hombre. Valores normales: insulina $<3 \mu \mathrm{U} / \mathrm{mL}$, péptido- $\mathrm{C}<0,6 \mathrm{ng} / \mathrm{ml}=0,2 \mathrm{nmol} / \mathrm{L}$ (2). * Los datos faltantes corresponden con exámenes no disponibles en práctica clínica diaria. Fuente: creación propia

\section{Estudios de localización}

Las pruebas de imagen no invasivas fueron realizadas en todos los casos como primer estudio para localizar el tumor. En orden de frecuencia se realizaron tomografía
( $\mathrm{n}=16 ; 84,2 \%)$, resonancia maognética nuclear (RMN) ( $\mathrm{n}=12 ; 63,1 \%$ ), gammagrafía con análogos de somatostatina $(\mathrm{n}=5 ; 26 ; 3 \%)$ y, PET 18FDG $(\mathrm{n}=1 ; 5,2 \%)$ (Tabla 2). 
Tabla 2. Métodos diagnósticos empleados para la localización de insulinoma

\begin{tabular}{lcc}
\hline \multicolumn{1}{c}{ Imagen } & $\mathbf{n}$ & $\begin{array}{c}\text { Casos positivos } \\
\mathbf{n}(\%)\end{array}$ \\
\hline Tomografía & 16 & $9(56)$ \\
Resonancia nuclear magnética & 12 & $10(83)$ \\
\hline Gammagrafía somatostatina & 5 & $3(60)$ \\
\hline PET 18FDG & 1 & $1(100)$ \\
\hline Ecografía endoscópica & 2 & $1(50)$ \\
\hline Arteriografía calcio & 4 & $2(50)$ \\
\hline Ecografía intraoperatoria* & 9 & $9(100)$ \\
\hline
\end{tabular}

* La ecografía intraoperatoria fue realizada en 2 pacientes como método para la localización del tumor y en 7 pacientes en quienes la lesión ya había sido localizada con otros métodos para planeación quirúrgica. Fuente: creación propia

La realización de las pruebas invasivas fue necesaria para la localización de la lesión en seis pacientes (31,5\%). Se practicó ecografía endoscópica en dos casos $(10,5 \%)$ y la prueba de estímulo intraarterial con calcio en cuatro pacientes (21\%). La ecografía intraoperatoria fue realizada en dos casos (10,5\%) como método para poder localizar de manera definitiva el tumor.

La imagen diagnóstica que identificó la localización del tumor con mayor frecuencia fue la RMN, en el $42,1 \%$ de los pacientes, seguido por la tomografía computarizada en el 31,5\%, la prueba de estímulo intraarterial con calcio en el 10,5 \% y ecografía endoscópica en solo uno (5,2 \%). Los métodos de localización prequirúrogicos permitieron la identificación del tumor en 17 de los 19 individuos (89,4\%), 2 de ellos $(10,5 \%)$ requirieron de localización intraoperatoria con ecografía y palpación.

En relación con los métodos no invasivos, la tomografía computarizada y la RMN fueron positivas en 56 y 83 \% de los casos, respectivamente. Se muestra en la Figura 1 las imágenes de localización de un paciente con insulinoma maliogno. La gammagrafía fue positiva en el $60 \%$ de los estudiados, pero solo fue usada en cinco casos del total y ya tenían localizado el tumor con otra imagen adicional. En relación con Ios métodos del diagnóstico invasivo, tanto la ecografía endoscópica como la prueba de estímulo intraarterial con calcio fueron positivas en el $50 \%$ de los casos, mientras que la ecografía intraoperatoria lo fue en el 100 \%. Se muestra en la Figura 2 el cateterismo selectivo de arterias pancreáticas con estímulo de calcio realizado en un insulinoma.
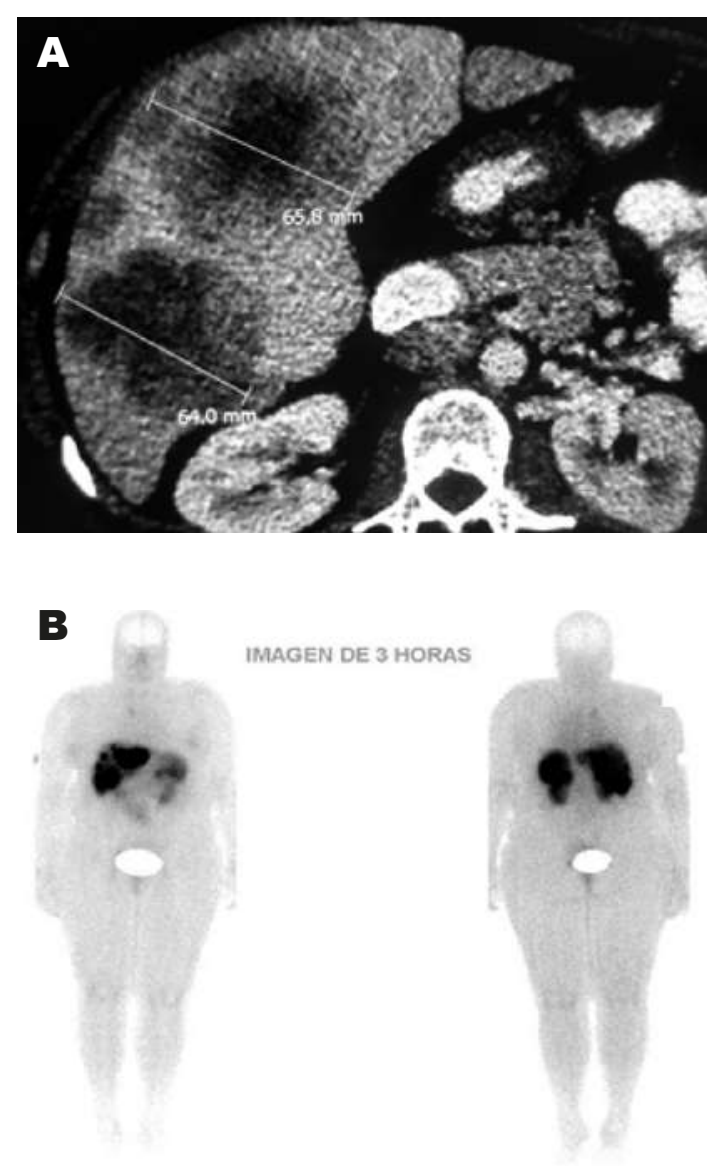

Figura 1. Imágenes de localización de insulinoma maligno en un paciente

Tomografía axial computarizada del abdomen. (A) gammagrafía con octreotide. (B). Mujer de 53 años con insulinoma maligno y multifocal de uno de los centros de referencia del estudio. Se resaltan las grandes y múltiples metástasis hepáticas. Fuente: creación propia 


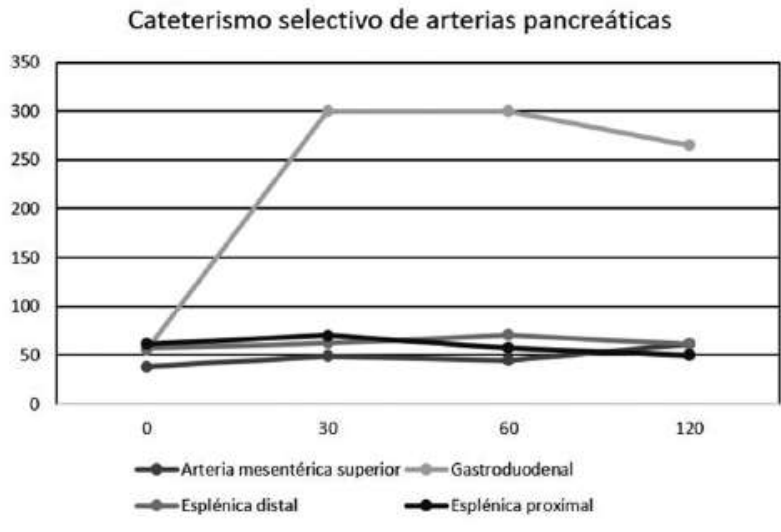

Figura 2. Cateterismo de arterias pancreáticas de un paciente del estudio Se evidencia el aumento de la insulina en la arteria gastroduodenal tras el estímulo con el calcio venoso. Fuente: creación propia

\section{Histopatología}

El promedio del mayor diámetro del tumor fue de 21,2 mm (D.E: 13,2 mm) (Tabla 3). La localización de las lesiones se distribuyó de la siguiente manera: cuatro casos localizados en la cabeza del páncreas (21\%), uno en el cuello (5,2 \%), tres en el proceso uncinado (15,7 \%), cuatro en el cuerpo (21\%), tres en la cola (15,7\%) y tres ocupaban múltiples territorios (15,7\%). Se encontraron cuatro casos de insulinomas múltiples (21\%) y dos de insulinoma maligno (10,5\%); el primer caso correspondió a una mujer de 53 años con metástasis hepáticas múltiples por insulinoma maligno con una histología de tumor neuroendocrino mal diferenciado y el segundo fue un hombre de 62 años, con una evolución rápida de los síntomas (dos meses) y múltiples metástasis hepáticas al momento del diagnóstico

El marcador de inmunohistoquímica Ki67 se realizó en nueve especímenes (50 \%) y, el índice mitótico se registró en 11 (61,1\%). De los casos con Ki67 reportados, seis tenían un valor menor a $3 \%(66,6 \%)$, dos un índice entre 3-20 \% (22,2 \%) y un caso más del 20 $\%(11,1 \%)$. Este último caso corresponde al paciente con insulinoma mal diferenciado mencionado anteriormente con un Ki67 de 40 \%. De los reportes con índice mitótico registrados, seis casos (54,5\%) tuvieron menos de dos mitosis en 50 CAP (campos de alto poder) $y$, cinco casos $(45,5 \%)$ turieron un índice entre
Tabla 3. Características histopatológicas de los insulinomas

\begin{tabular}{|c|c|}
\hline Características & n (\%)* \\
\hline \multicolumn{2}{|l|}{ Localización } \\
\hline Cabeza & $4(22,2)$ \\
\hline Cuello & $1(5,5)$ \\
\hline Proceso uncinado & $3(16,6)$ \\
\hline Cuerpo & $4(22,2)$ \\
\hline Cola & $2(11,1)$ \\
\hline Más de una localización & $4(22,2)$ \\
\hline \multicolumn{2}{|l|}{ Focalidad } \\
\hline Única & $14(77,7)$ \\
\hline Múltiple & $4(20)$ \\
\hline \multicolumn{2}{|l|}{ Maligno } \\
\hline Sí & $2(11,1)$ \\
\hline No & $16(88,9)$ \\
\hline \multicolumn{2}{|l|}{ Estadio TNM } \\
\hline I & $11(61,1)$ \\
\hline II & $6(33,3)$ \\
\hline III & $0(0)$ \\
\hline IV & $1(5,6)$ \\
\hline \multicolumn{2}{|l|}{ Clasificación OMS 2017} \\
\hline \multicolumn{2}{|l|}{ Bien diferenciado } \\
\hline G1 & $6(33,3)$ \\
\hline G2 & $5(27,7)$ \\
\hline G3 & $0(0)$ \\
\hline Mal diferenciado & $1(5,6)$ \\
\hline Sin dato & $6(33,3)$ \\
\hline
\end{tabular}

* Se incluyó a 18 pacientes con patología quirúrgica, uno con insulinoma maligno no tuvo intervención quirúrgica. Fuente: creación propia

dos y 20 mitosis en 50 CAP No hubo ningún caso de los analizados con más de 20 mitosis en 50 CAP.

Según la clasificación de la Organización Mundial de la Salud del 2017, para 13 casos analizados hubo: un caso con tumor mal diferenciado y 12 casos con tumores bien diferenciados; entre ellos siete fueron grado I y cinco grado II; no se presentó ningún caso de tumor neuroendocrino bien diferenciado grado III (Tabla 3).

\section{Tratamiento}

El tratamiento quirúrgico se realizó en 18 individuos (94,7 \%) (Tabla 4). En un paciente no se realizó 
intervención quirúrogica por el diagnóstico de insulinoma maligno. La cirugía fue abierta en 10 (55,5\%) y laparoscópica en 8 (44,4\%). El tipo de ciruớa más frecuentemente realizada fue la pancreatectomía subtotal ( $\mathrm{n}=7 ; 38,8 \%$ ), seguida de la enucleación (n $=4 ; 22,2 \%)$, pancreatectomía distal $(n=4 ; 22,2 \%)$ y, en último lugar, la cirugía de Whipple $(\mathrm{n}=3 ; 16,6 \%)$. Las complicaciones quirúroicas ocurrieron en nueve individuos (50\%), siendo las más frecuentes la sepsis de origen abdominal y la insuficiencia endocrina (cada una con frecuencia de 16,6\%).

El tratamiento médico se administró en tres pacientes con insulinomas (15,7\%), uno recibió diazóxido, otro octreotide y el tercero correspondiente con un caso de insulinoma maliogno que fue tratado inicialmente con diazóxido y octreotide, luego, con cisplatino más etopósido y dos sesiones de lutecio. Las sesiones faltantes de lutecio no fueron autorizadas por su seguridad social por trámites administrativos. Posteriormente recibió everolimus con control clínico de la hipoglucemia.

Tabla 4. Tratamiento y complicaciones

\begin{tabular}{lc}
\hline \multicolumn{1}{c}{ Características } & n $(\%)$ \\
Abordaje quirúrgico & $10(55,5)$ \\
\hline Abierto & $8(44,4)$ \\
\hline Laparoscópico & \\
Tipo de cirugía & $4(22,2)$ \\
\hline Enucleación & $4(22,2)$ \\
\hline Pancreatectomía distal & $7(38,9)$ \\
\hline Pancreatectomía subtotal & $3(16,7)$ \\
\hline Whipple & \\
\hline Complicaciones quirúrgicas & $1(5,6)$ \\
\hline Fístula pancreática & $3(16,6)$ \\
\hline Infección & $1(5,6)$ \\
\hline Hemorragia & $1(5,6)$ \\
\hline Pancreatitis & $3(16,6)$ \\
\hline Insuficiencia endocrina & $1(5,6)$ \\
\hline Insuficiencia exocrina & $3(16,6)$ \\
\hline Muerte & $1(5,6)$ \\
\hline Otra & $2(11,1)$ \\
\hline Tratamiento médico & $2(11,1)$ \\
\hline Diazóxido & $1(5,6)$ \\
\hline Análogo de somatostatina & \\
\hline Otro & \\
\hline
\end{tabular}

Fuente: creación propia

\section{Evolución y seguimiento}

La mediana de estancia hospitalaria fue de 23,5 días (RIC 12-32). La mortalidad fue de $15,8 \%$ en el posoperatorio temprano, derivada de las complicaciones posoperatorias, relacionadas con una infección. Los sobrevivientes tuvieron una curación del $100 \%$ en el seguimiento. Hasta febrero del 2017 se logró comprobar el estado vital mediante llamadas telefónicas de 10 individuos, todos estaban vivos (52,6\%). En 5 no se logró contacto telefónico. La mediana de supervivencia fue de 50 meses (RIC 14-62).

\section{DISCUSIÓN}

El enfoque diagnóstico del insulinoma se basa en la demostración de hipooglucemia e hiperinsulinismo endógeno mediante pruebas bioquímicas que dan lugar a la realización de imágenes convencionales como la tomografía y RMN, para en la mayoría de los casos, localizar el origen del exceso hormonal y luego proceder a realizar una intervención quirúrgica con el fin de extirpar el tejido hiperfuncionante.

En el presente estudio se presentaron Ias características clínicas, los métodos diagnósticos, los resultados de histopatología y el tratamiento en 19 pacientes con insulinoma en tres hospitales de alto nivel de complejidad. Esta es la serie más grande publicada a la fecha en Colombia.

Se confirma una discreta predilección por el sexo femenino (52,6\%), con una relación mujer/hombre de 1,1:1. El promedio de edad del diagnóstico fue similar a Io descrito en la Clínica Mayo ${ }^{(1)}$. Interesantemente, los casos con NEM-1 no tuvieron una edad más temprana en el diagnóstico comparada con lo descrito ${ }^{(1,10)}$, dado que usualmente, son diagnosticados entre los 15 a 18 años antes que los casos esporádicos.

Las características del insulinoma en esta serie se corresponden con tumores únicos, esporádicos y benignos en su gran mayoría ${ }^{(3,11)}$. En comparación con lo reportado por la Clínica Mayo en Estados Unidos, nuestros datos muestran un porcentaje mayor de insulinomas malignos, 10,6 \% comparado $4 \%$, un porcentaje mayor de insulinoma asociado a NEM-1, 15,8 $\%$ vs. $6 \%$ y, una mayor frecuencia de insulinoma multifocal $21,1 \%$ vs $17 \%{ }^{(3)}$. 
La mediana del tiempo de evolución de los síntomas (11 meses) no difiere de lo descrito, a pesar de las frecuentes dificultades de acceso al sistema de salud ${ }^{(3,12)}$. Todos presentaron hipoglucemia en ayunas y, un 63,2 $\%$ estuvo asociado con la hipoglucemia posprandial. Hubo una clara predominancia de los síntomas neuroglucopénicos sobre los autonómicos, concordante con otras series ${ }^{(4,13)}$. Asimismo, se identificó que las personas con insulinoma tenían un diagnóstico errado de epilepsia o de trastorno psiquiátrico, lo cual conduce a la reflexión de la importancia de considerar una hipoglucemia en estos casos.

El tiempo a la presentación de hipoglucemia durante la prueba de ayuno fue $<48$ horas en el $100 \%$ y, $92,3 \%$ tuvieron hipoglucemia en las primeras 24 horas. Esta observación contrasta con lo reportado por la Clínica Mayo en Estados Unidos, en la que un 14 \% de los pacientes desarrollaron hipoglucemia después de las 48 horas ${ }^{(14)}$.

En nuestro estudio el diagnóstico bioquímico fue realizado con insulina elevada en todos Ios casos, pero el péptido C fue reportado en solo el 47,3\%. La prueba de sulfonilureas se realizó en un caso y los cuerpos cetónicos fueron ordenados en siete individuos $(36,8 \%)$. Las limitaciones locales que detectamos para acceder a las pruebas de diagnóstico bioquímico fueron: 1. El péptido $C$ es un estudio realizado en grandes laboratorios de referencia y con tiempo de reporte del resultado prolongado, usualmente, superior a una semana. 2. Los niveles de sulfonilureas, por razones de alto costo, no son autorizadas por el sistema de salud. 3. El B-hidroxibutirato es el cuerpo cetónico de elección para el estudio de hipoglucemia, no está disponible en el medio local y solo podemos medir cuerpos cetónicos totales. 4. Los anticuerpos antiinsulina, que se recomiendan de rutina para excluir hipoglucemia autoinmune como diagnóstico diferencial, no se realizan de manera rutinaria bajo la consideración de la baja prevalencia de esta entidad en la población latinoamericana y 5 . La prueba de glucagón no se realizó en ninguno de los casos; su uso no se encuentra incluido en ninguno de los protocolos de la prueba de ayuno de las instituciones, la razón más probable es la difícil autorización al no tener registro Invima para hipoglucemia no diabética.

Desde el punto de vista de localización, la mayoría de los pacientes necesitaron más de una técnica de imaggen para el diagnóstico, solo el 31,5\% de los casos tuvieron un diagnóstico con la prueba imaginológica inicial. Sin embargo, en la mayoría de los casos $(89,4 \%)$ se logró la localización preoperatoria de la lesión. La capacidad de detección por la RMN fue claramente superior a la de la tomografía ( $83 \%$ vs. $56 \%$ ), por ende, esta debería ser la imagen inicial de estudio en insulinoma. El rendimiento de estas pruebas de imagen es variable.

EI PET 18FDG fue el método diagnóstico preoperatorio con mejor desempeño diagnóstico, detectando el $100 \%$ de las lesiones. Sin embargo, este resultado debe ser analizado con cautela, dado que se trata de un insulinoma maliono, en el cual este estudio fue realizado con motivo de estadificación.

En los pacientes con estudios imaginológicos negativos, las pruebas invasivas lograron la localización preoperatoria del tumor en el $50 \%$ de los casos, sin diferencias en el rendimiento diagnóstico entre ecografía endoscópica y la prueba de estímulo intraarterial con calcio. Sin embargo, el bajo rendimiento de estos dos métodos (50\% para ambos), contrasta con la alta sensibilidad ( $>80 \%$ ) reportada en la literatura ${ }^{(15-18)}$. No se describe en el estudio las técnicas de medicina nuclear de última generación, tales como el PET con galio-68, que tiene reportada en la literatura una sensibilidad entre el $82-100 \%$, debido a que a la fecha del reporte, esta técnica no está disponible en el medio local ${ }^{(19)}$.

La ecografía intraoperatoria se comportó como un método con excelente rendimiento diagnóstico, con detección de un $100 \%$ de los tumores. Este hallazogo denota la experiencia local con el uso de este método, tanto para localización intraoperatoria en personas con estudios previos negativos como en la decisión de la técnica quirúrogica en medio del procedimiento.

La localización anatómica de las lesiones no tuvo predilección por alguna región específica del páncreas. Se identificaron 3 casos de insulinoma maliono, de los cuales solo uno presentó características histopatológicas del tumor neuroendocrino mal diferenciado.

En 12 casos (66\%), el informe de histopatología permitió categorizar el tumor según la clasificación OMS 2017 para tumores neuroendocrinos pancreáticos y, en los casos restantes, hubo un reporte incompleto de las características histopatológicas o 
inmunohistoquímicas. Este hecho sugiere una baja experiencia en el medio para el reporte de la patología de tumores neuroendocrinos, considerando su baja frecuencia. Además, puede ser un reflejo de las barreras económicas que existen en el medio para la realización de rutina de una inmunohistoquímica completa.

Se practicó un abordaje laparoscópico en el 44,4 \% de los casos, proporción superior a lo reportado en otras series hispanoamericanas: 29 \% en una serie argentina de 37 pacientes $^{(11)}$ y 7,4 \% en una española de 27 insulinomas ${ }^{(12)}$. Nuestros datos muestran que se realizó enucleación en el 22,2 \%, loogrando así la curación en el $100 \%$ de los casos. La insuficiencia pancreática endocrina fue la principal complicación posquirúrgica, presente en el 16,7 \% y, la fístula pancreática, reportada en la mayoría de las series como la primera complicación en frecuencia, se presentó solo en 1 caso $(5,2 \%)^{(20)}$.

Todos los casos de mortalidad $(\mathrm{n}=3)$ fueron relacionados con complicaciones quirúrogicas, a diferencia de lo reportado en la literatura, en donde la mortalidad es descrita a mediano y largo plazo en relación directa con la presencia de enfermedad metastásica (21). La mayor frecuencia de complicaciones asociadas con la cirugía podría estar relacionada con el bajo volumen quirúrogico de insulinoma en nuestros centros hospitalarios, inherente a un tumor de presentación rara, en comparación con el alto volumen de casos reportado en los grandes centros del mundo.

Las principales limitaciones de este trabajo son propias del tipo de estudio, ambispectivo y multicéntrico, lo cual implica ausencia de algunos datos, falta de homogeneidad en los criterios diagnósticos, en la experiencia con realización de las pruebas de localización y en las conductas quirúroicas y sus desenlaces.

En conclusión, las características demográficas y la presentación clínica del insulinoma en nuestro medio son similares a las descritas en la literatura. Existen grandes limitaciones relacionadas con la disponibilidad de algunas pruebas diagnósticas, en particular, con la medición de sulfonilureas. El rendimiento diagnóstico de las técnicas de imagen destaca a la RNM como un método sensible en el abordaje inicial y señala un menor desempeño de los métodos preoperatorios de localización invasiva.

\section{CONFLICTOS DE INTERESES}

Ninguno por declarar.

\section{REFERENCIAS BIBLIOGRÁFICAS}

1. Service FJ, McMahon MM, O’Brien PC, Ballard DJ. Functioning insulinoma--incidence, recurrence, and long-term survival of patients: a 60-year study. Mayo Clin Proc. 1991;66(7):711-9. DOI 10.1016/s00256196(12)62083-7.

2. Cryer PE, Axelrod L, Grossman AB, Heller SR, Montori VM, Seaquist ER, et al. Evaluation and management of adult hypoglycemic disorders: an Endocrine Society Clinical Practice Guideline. J Clin Endocrinol Metab. 2009;94(3):709-28. DOI 10.1210/jc.2008-1410.

3. Placzkowski KA, Vella A, Thompson GB, Grant CS Reading CC, Charboneau JW, et al. Secular trends in the presentation and management of functioning insulinoma at the Mayo Clinic, 1987-2007. J Clin Endocrinol Metab. 2009;94(4):1069-73. DOI 10.1210/ jc. 2008-2031.

4. Dizon AM, Kowalyk S, Hoogwerf BJ. Neuroglycopenic and other symptoms in patients with insulinomas. Am J Med. 1999; 106(3):307-10.

5. Falconi M, Eriksson B, Kaltsas G, Bartsch DK, Capdevila J, Caplin M, et al. ENETS Consensus Guidelines Update for the Management of Patients with Functional Pancreatic Neuroendocrine Tumors and Non-Functional Pancreatic Neuroendocrine Tumors. Neuroendocrinology. 2016;103(2):153-71. DOI $10.1159 / 000443171$.

6. Giraldo JA, Melo M, Abadía M, Pantoja D, Garavito G, Mesa J, et al. Insulinoma del páncreas: reporte de tres casos y discusión. Rev Col Gastroenterol. 2008;23(3):280-8.

7. De la Hoz J, Marulanda S. Insulinoma. Experiencia con 8 casos operados y revisión del tema. Rev Col Cir. 1995;10(2):63-78.

8. Jácome Roca A, Arango Restrepo G. Insulinoma. Análisis de cinco casos. Rev Asoc Col Endocrinol Diab. 1982;13(3):106-18.

9. González-Clavijo AM, Fierro-Maya LF. Patient with neuropsychiatric symptoms and insulinoma of difficult preoperative localization. Rev. Fac. Med. 2014;62(4):637-40. 
10. Toaiari M, Davì MV, Dalle Carbonare L, Boninsegna L, Castellani C, Falconi M, et al. Presentation, diagnostic features and glucose handling in a monocentric series of insulinomas. J Endocrinol Invest. 2013;36(9):753-8.

11. Diaz AG, Lucas S, Ferraina P, Ferraro A, Puchulu F, Paes De Lima A, et al. [Clinical experience in 37 cases of insulinoma]. Medicina (B Aires). 2006;66(6):499-504.

12. Iglesias P, Lafuente C, Martín Almendra M, López Guzmán A, Castro JC, Díez JJ. Insulinoma: a multicenter, retrospective analysis of three decades of experience (1983-2014). Endocrinol Nutr. 2015;62(7):306-13. DOI 10.1016/j.endoen.2015.08.006.

13. Tavcar I, Kiković S, Bezmarević M, Rusović S, Perisić $\mathrm{N}$, Mirković D, et al. A 60-year expirience in the treatment of pancreatic insulinoma in the Military Medical Academy, Belgrade, Serbia. Vojnosanit Pregl. 2014;71(3):293-7. DOI 10.2298/VSP130415048T.

14. Service FJ, Natt N. The prolonged fast. J Clin Endocrinol Metab. 2000;85(11):3973-4. DOI 10.1210/ jcem.85.11.6934.

15. Mehrabi A, Fischer L, Hafezi M, Dirlewanger A, Grenacher L, Diener MK, et al. A systematic review of localization, surgical treatment options, and outcome of insulinoma. Pancreas. 2014;43(5):675-86. DOI 10.1097/MPA.0000000000000110.

16. Mirallié E, Pattou F, Malvaux P, Filoche B, Godchaux JM, Maunoury $\nabla$, et al. [Value of endoscopic ultrasonography and somatostatin receptor scintigraphy in the preoperative localization of insulinomas and gastrinomas. Experience of 54 cases]. Gastroenterol Clin Biol. 2002;26(4):360-6.

17. Pereira PL, Roche AJ, Maier GW, Huppert PE, Dammann F, Farnsworth CT, et al. Insulinoma and islet cell hyperplasia: value of the calcium intraarterial stimulation test when findings of other preoperative studies are negative. Radiology. 1998;206(3):703-9. DOI 10.1148/radiology.206.3.9494488.

18. Rösch T, Lightdale CJ, Botet JF, Boyce GA, Sivak MV, Yasuda K, et al. Localization of pancreatic endocrine tumors by endoscopic ultrasonography. N Engl J Med. 1992;326(26):1721-6. DOI 10.1056/ NEJM199206253262601.

19. Guzmán YF, López RdP, Vera A, González-Devia D. Herramientas para el abordaje diagnóstico de los tumores neuroendocrinos de páncreas. Rev Colomb Cir. 2018;33:79-99. DOI 10.30944/20117582.50.

20. Finlayson E, Clark OH. Surgical treatment of insulinomas. Surg Clin North Am. 2004;84(3):775-85. DOI 10.1016/j.suc.2004.02.004.

21. Câmara-de-Souza AB, Toyoshima MTK, Giannella ML, Freire DS, Camacho CP, Lourenço DM, et al. Insulinoma: A retrospective study analyzing the differences between benign and malignant tumors. Pancreatology. 2018;18(3):298-303. DOI 0.1016/j.pan.2018.01.009. 